\title{
Enseñar los juicios de Núremberg en un centro penitenciario. Una propuesta educativa
}

Teaching the Nuremberg Trials in a penal institution. An educational proposal

Miguel Ángel Pallarés Jiménez*, Sergio Sánchez Martínez*

\section{Resumen}

Los Centros Penitenciarios poseen módulos educativos donde se procura dotar de enseñanza reglada a los internos para favorecer su reinserción, como se recoge en la Constitución Española de 1978 así como en los decretos de Enseñanza Secundaria para personas adultas (ESPA). En este marco, se ofrece aquí una propuesta educativa para desarrollar un tema controvertido, como es el del Holocausto, en un ámbito educativo especialmente sensible a procesos educativos en situaciones de confinamiento, un aula de 4ำ de ESPA en un centro penitenciario, a través del conocimiento de las responsabilidades criminales consideradas en los Juicios de Núremberg. Utilizando la representación dramática como recurso didáctico, ya que los internos tendrían que reproducir los distintos roles de los participantes en los juicios, se pretende, en las diferentes sesiones en las que está estructurada esta propuesta, tratar de las responsabilidades morales, entender la necesaria existencia de unas instituciones fuertes con códigos de convivencia adecuados y desarrollar la empatía con las víctimas del régimen nazi, con el objetivo de que sean desarrollados valores cívicos entre los internos

Palabras clave: Didáctica de las Ciencias Sociales. Didáctica en Centros Penitenciarios. Educación de Adultos. Educación en Centros Penitenciarios. Holocausto.

\begin{abstract}
The penal institutions have educational modules where the inmates are provided with regulated education to favor their reinsertion, as stated in the Spanish Constitution of 1978, as well as in the Secondary Education decrees for adults (ESPA). Within this framework, an educational proposal is offered here to develop a controversial subject, such as the Holocaust, in an educational environment that is especially sensitive to educational processes in situations of confinement, a 4th grade ESPA classroom in a penitentiary center, through the knowledge of the responsibilities emanating from the Nuremberg Trials. Using dramatic representation as a didactic resource, since the inmates would have to reproduce the different roles of the participants in the trials, it is intended, in the different sessions in which this proposal is structured, to deal with moral responsibilities, to understand the necessary existence of strong institutions with adequate codes of coexistence, and to develop empathy with the victims of the Nazi regime, with the aim of developing civic values among the inmates.
\end{abstract}

Key words: Didactics of Social Sciences. Didactics in penal institutions. Adult education. Education in penal institutions. Holocaust.

\footnotetext{
* Universidad de Zaragoza, Grupo de investigación ARGOS, IUCA • M. A. Pallarés Jiménez (ORCID 0000-0003-3459-5965). S. Sánchez Martínez (ORCID 0000-0002-6382-8142) • Autor de correspondencia: Miguel Ángel Pallarés Jiménez (miguelap@unizar.es).
}

Pallarés Jiménez, M. A. y Sánchez Martínez, S. (2020). Enseñar los juicios de Núremberg en un centro penitenciario. Una propuesta educativa. CLIO. History and History teaching, 46, 122-135. https://doi.org/10.26754/ojs clio/clio.2020465293. Recibido: 10/9/2020. Aceptado: 4/12/2020. 


\section{Introducción}

Tras la Segunda Guerra Mundial urgía la necesidad de juzgar a los responsables de las atrocidades cometidas. Las potencias vencedoras plantearon diversas formas de imponer justicia a los principales líderes y organizaciones, sobre todo de Alemania; de esta manera, se produjeron fuertes debates entre los aliados con el objetivo de fijar cuál era el procedimiento más adecuado para castigar a los criminales de guerra nazis, desde las iniciales propuestas británicas, de llevar a cabo penas capitales inmediatas, hasta las propuestas estadounidenses y soviéticas, de realizar juicios con garantías en un formato mediático y popular (Overy, 2003, p. 27 y ss.).

Estas opciones últimas fueron las que finalmente se impusieron, y según el Acuerdo de Londres, del 8 de agosto de 1945, se estableció el Tribunal Militar Internacional de Núremberg, que había de juzgar a los jerarcas de la dictadura hitleriana. Su artículo 6 había de inspirar posteriormente las normas que se ocupan del genocidio, los crímenes de lesa humanidad y los crímenes de guerra, recogidas en el vigente Estatuto de Roma, del 17 de julio de 1998, por el cual se creó la Corte Penal Internacional de carácter permanente.

El paso dado tras dicha guerra ha sido considerado por el jurista Antonio Cassese "monumento importantísimo de la civilización jurídica moderna", a pesar de la polémica provocada por el quebrantamiento del principio de legalidad: nadie puede ser castigado por una acción que, en el momento de llevarse a cabo, no se consideraba criminal; de hecho, la comunidad internacional de derechos humanos ha justificado el proceso de Núremberg por tres razones fundamentales: porque la punibilidad de las atrocidades imputables al regimen nazi estaba sustentada en el derecho natural y en el consuetudinario; porque se trataba de evitar el mal mayor de la impunidad; y, sobre todo, porque la novedad radical de los crímenes del nazismo, y en especial el genocidio de los pueblos judío y gitano, exigía una respuesta judicial inédita. De esta manera, la invención de la responsabilidad penal internacional del individuo, por el llamado "derecho de Núremberg", es la base de la nueva jurisdicción penal internacional, que constituye un verdadero avance de la razón democrática y humanitaria; no obstante, el establecimiento de esta justicia de la humanidad no sería posible sin la consagración normativa de los crímenes graves contra el derecho internacional publico, que hoy se agrupan en tres categorías principales: crímenes de 
lesa humanidad, incluido el genocidio; crímenes de guerra o infracciones graves al derecho internacional humanitario; y crímenes contra la paz (Valencia, 2000, p. 85).

Sin duda Núremberg, junto al posterior juicio a Adolf Eichmann en Jerusalén (Arendt, 1999), determinaron los patrones clave para que los criminales de guerra no quedaran sin juicio ni castigo; ambos fueron mediáticos, ambos tuvieron un eco posterior notable, además de establecer la tipificación de delitos que no tenían parangón en la Historia y que, por desgracia, se repitieron en las guerras de desintegración de la antigua Yugoslavia y en otros enfrentamientos fuera de Europa; delitos contra la humanidad y crímenes contra poblaciones enteras, genocidio y Holocausto, fueron nuevas y desgraciadas palabras que surgieron en un siglo $\mathrm{XX}$ especialmente devastador (Temon, 1995).

Poco después de los juicios de Núremberg, el 9 de diciembre de 1948, la Asamblea General de las Naciones Unidas aprobó la resolución 260 A (III) para la prevención y castigo del delito de genocidio, que no era otra cosa que la destrucción o la tentativa de destrucción intencionada, en todo o en parte, de un grupo nacional, étnico, racial o religioso; lo que se reprodujo textualmente en el artículo 6 del Estatuto de Roma. En palabras de Hannah Arendt, el genocidio constituye el crimen de los crímenes porque pretende destruir la diversidad humana (Valencia, 2000, pp. 87-88).

Se pretendía trabajar en clase un tema tan controvertido como es el del Holocausto (Hayes and Roth, 2010), que se viene considerando desde los cursos finales de Educación Primaria (Gray, 2015), y así lo recoge el currículo en España ${ }^{1}$; pero en un ámbito pedagógico especialmente sensible a procesos educativos en situaciones de confinamiento, un aula de $4^{\circ}$ de ESPA en un centro penitenciario, a través del conocimiento de las responsabilidades criminales consideradas en los Juicios de Núremberg, así como de su desarrollo, su contexto histórico y su marco legal; haciendo hincapié en el reconocimiento de valores, obvios desde el punto de vista jurídico y necesarios desde el punto de vista social. Se pretendía recalcar la importancia de las dificultades que hubo que solventar en la preparación y desarrollo de los juicios de los más importantes dirigentes nazis; y, a través de diversas actividades, se abordaría el enfoque de las distintas comisiones que se organizaron en

\footnotetext{
1 Real Decreto 126/2014, de 28 de febrero, por el que se establece el currículo básico de Educación Primaria (BOE, nำ52, de 01/03/2014).
} 
1945 para juzgar a los reos, de manera que fueran presentados a los alumnos parte de los dilemas que debieron superar los fiscales aliados, procurando que fueran los propios alumnos quienes tomaran durante el ejercicio las decisiones más pertinentes. Por otro lado, ellos también realizarían una representación del desarrollo del juicio contra los criminales de guerra, barajando de forma resumida las acusaciones, pruebas y veredictos; lo que no dejaría de ser paradójico, en un espacio carcelario. Finalmente, se optaría por la visualización de la película Núremberg (Simoneau, 2000), con un posterior debate.

Durante el curso 2019-2020, se consideró que podía ser una actividad conveniente a desarrollar en el Centro Penitenciario de Zuera, sito en la localidad zaragozana homónima, con los alumnos internos matriculados en la Enseñanza Secundaria para Personas Adultas (ESPA). Aunque los problemas acarreados por la Covid-19 no permitieron que la propuesta se llevara a cabo, se muestran aquí los objetivos didácticos y el planteamiento para su realización, que no solo tendría validez en el contexto de un centro penitenciario, sino en cualquier centro educativo de Secundaria, si así se deseara.

Ante todo, procede resumir varias cuestiones de contextualización para comprender mejor su diseño, siendo necesario destacar la escasísima bibliografía existente sobre didáctica de las Ciencias Sociales en entornos penitenciarios, dado que la mayor parte de la producción científica ha profundizado en los centros penitenciarios sobre cuestiones pedagógicas, desde la perspectiva de la psicología social. Es por ello que resultan necesarias contribuciones como esta, para destacar la importancia que puede adquirir la didáctica en entornos educativos no pertenecientes a un instituto de enseñanza Secundaria ${ }^{2}$.

\section{El contexto educativo: la enseñanza Secundaria en el centro penitenciario de}

\section{Zuera}

En primer lugar es necesario contextualizar varios elementos y factores que determinan la docencia en un centro penitenciario. La Constitución Española de 1978 establece en su artículo 27 el derecho a la educación para "todos". Además, en el

\footnotetext{
${ }^{2}$ El entorno educativo penitenciario de Secundaria es probable que tampoco haya tenido un marco legal y administrativo muy definido (véase, para el caso de Cataluña, Beatriz, 2013, pp. 331-337).
} 
artículo 25.2, relacionado con la reclusion, recoge: "Las penas privativas de libertad y las medidas de seguridad estarán orientadas hacia la reeducación y reinserción social" garantizando el "acceso a la cultura y al desarrollo integral de su personalidad". Partiendo del ordenamiento jurídico superior, se desarrollan las leyes educativas pertinentes correspondientes a la Administración del Estado y a las Comunidades Autónomas; es pues la LOMCE quien regula la enseñanza de educación de adultos y, en nuestro caso, el decreto 1693/2019 del Gobierno de Aragón el que determina el currículo y el funcionamiento de tales enseñanzas, que se puede considerer muy novedoso.

Nuestro centro penitenciario albergaba a comienzos de 2019 unos 1.200 internos aproximadamente ${ }^{3}$; está dividido en 16 módulos, de los cuales uno es exclusivo de mujeres. La población reclusa se distribuye en dichos módulos en función de sus delitos: reincidencia, terapias, trabajo dentro de la prisión, etc.; por lo que existen múltiples variables para determinar el módulo donde el interno pasará su privación de libertad. Dentro del centro existe un módulo sociocultural donde se imparten talleres, cursos diversos, se realizan conciertos o actividades polivalentes (dado que posee un salón de actos completo), biblioteca penitenciaria y, por supuesto, aulas para impartir la docencia. En Zuera la enseñanza de adultos reglada se divide en dos ramas: por un lado, la Formación Inicial para Personas Adultas (FIPA) y, por otro, la enseñanza Secundaria (ESPA). Los niveles de FIPA se subdividen en Alfabetización, Neolectores y Consolidación; además, se encarga de impartir español para extranjeros en varios niveles. ESPA se divide en dos niveles con dos módulos cada uno ( $1^{\circ}$ y $2^{\circ}$, y $3^{\circ}$ y $\left.4^{\circ}\right)$, casi correspondientes con los niveles de la ESO. Los alumnos cursan los niveles por cuatrimestres, pudiendo en un curso académico realizar los dos primeros módulos ( $1^{\circ}$ y $2^{\circ}$ de ESPA) y en el siguiente curso los restantes ( $3^{\circ}$ y $4^{\circ} \stackrel{0}{ }$ de ESPA). En cada uno de los módulos existen diversos bloques establecidos en ámbitos (Comunicación, Social y Científico-Tecnológico), cada uno de ellos con sub ámbitos que reflejan la correspondencia con las materias ${ }^{4}$. A nivel orgánico, en el caso del centro

\footnotetext{
${ }^{3}$ Se pueden consultar estadísticas más precisas en la página web de Instituciones Penitenciarias: www.institucionpenitenciaria.es, así como cualquier otra información relevante que complete lo expuesto en este texto.

${ }^{4}$ Por ejemplo, un alumno de centro penitenciario que cursa el primer nivel, primer módulo, ámbito social, sub ámbito de Geografía e Historia; correspondería, aproximadamente, a un nivel de 1ํ de ESO.
} 
penitenciario no representa una unidad autónoma, sino que es un "aula externa" dependiente del Centro de Educación de Adultos Margen Izquierda de Zaragoza ${ }^{5}$.

Respecto al número de alumnos interesados en matricularse en la enseñanza reglada, oscila entre unas 400 a 600 solicitudes por curso6. Para ingresar en la ESPA se realiza una prueba inicial de nivel para determinar los conocimientos previos; además, los internos pueden aportar documentación de cursos reglados realizados para poder continuar con los estudios, tanto de otro centro penitenciario como de otros centros de educación.

Como punto final diremos que la formación reglada de internos en situación de privación de libertad, supone un reto para el docente y también para el alumnado. A las particularidades de la enseñanza de adultos, se suman las historias individuales de cada interno, presentándose la suma de todos esos factores para el profesor como un aliciente para realizar la acción educative; es por ello que, actividades que se salgan de las rutinas tradicionales en la metodología diaria, resultan especialmente atractivas y motivadoras para los alumnos, de manera que los objetivos docentes y los efectos transversales de esta actividad, enfocados a la educación en valores, hubieran sido especialmente adecuados para su desarrollo.

Así pues, la clase a la que iba dirigida la actividad (4ํㅡódulo de ESPA) está formada por 12 alumnos, de los cuales cuatro promocionan del tercer módulo y el resto han llegado nuevos al centro penitenciario. Se debe tener en cuenta las dificultades propias del centro penitenciario, pues los alumnos a veces se ausentan de las clases por múltiples motivos (comunicaciones, llamadas telefónicas, asistencia a juicios, etc.); con todo, se conformó un bloque homogéneo de alumnos que asistían regularmente. También es importante destacar que en el módulo $4^{\circ}$ de ESPA las sesiones son de dos días a la semana, de dos horas continuadas cada día.

\footnotetext{
${ }^{5}$ Para más información, véase la web: http://www.cpepamargenizquierda.com/

${ }^{6}$ Dichas cifras emanan del número de solicitudes recibidas en el módulo socio-cultural para ingresar en la enseñanza reglada. La fuente de las cifras es Sergio Sánchez Martínez.
} 


\section{Currículo: el Holocausto y el juicio de Núremberg en la educación para adultos. Objetivos didácticos. Educación en valores}

La orden de currículo de ESPA (Gobierno de Aragón, 2019), establece en su módulo cuarto, sub módulo de sociales, la Historia contemporánea tanto mundial como española. En concreto, en el bloque 6, "las causas y consecuencias de la Segunda Guerra Mundial (1939-1945)", en cuyos contenidos a tratar aparecen específicamente tanto el proceso de guerra europea a guerra mundial, como el Holocausto. Es pues en este contexto curricular donde se sitúa nuestra actividad y, junto con los contenidos, el criterio de evaluación correspondiente:

“Crit.AMS.6.2. Evaluar el Holocausto, relacionándolo con el contexto ideológico de las fuerzas políticas que lo llevaron a cabo y que causaron la guerra europea, valorando sus consecuencias. Avanzar en la consecución de memoria democrática para adoptar actitudes que intenten evitar que esas situaciones se vuelvan a producir". (Gobierno de Aragón, 2019).

Las Competencias Básicas son las de Comunicación Lingüística, y Social y Ciudadana; y el estándar de aprendizaje, el Est.AMS.6.2.1., que "Reconoce el significado del Holocausto en la historia mundial para evitar que hechos similares se vuelvan a repetir". Respecto a los temas transversales, referimos aquel que recoge el decreto de currículo:

(...) "los valores que sustentan la libertad, la justicia, la igualdad, el pluralismo político, la paz, la democracia, el respeto a los derechos humanos y el rechazo a la violencia terrorista, la pluralidad, el respeto al Estado de derecho, el respeto y consideración a las víctimas del terrorismo, y la prevención del terrorismo y de cualquier tipo de violencia".

Los juicios de Núremberg no aparecen mencionados expresamente en el decreto de currículo, aunque consideramos que representan una de las piezas claves para la comprensión del Holocausto. Los conocimientos previos para la actividad resultan determinantes, sobre el desarrollo de los contenidos sobre la Segunda Guerra Mundial y el Holocausto: se trata de concluir, a modo de síntesis activa por parte de los alumnos, la capacidad de determinar las responsabilidades de los actores y las organizaciones implicadas en el genocidio. 
Por otro lado, se establecen unos objetivos pormenorizados para la actividad, definiéndose de la siguiente forma:

Obj. 1.- Determinar las responsabilidades en individuos y organizaciones para la ejecución del programa alemán de exterminio.

Obj. 2.- Decidir la gravedad de los hechos probados mediante la documentación y las fuentes facilitadas.

Obj. 3.- Investigar y tomar conciencia de los delitos cometidos por los implicados.

Obj. 4.- Valorar el respeto a la vida y la tolerancia como fundamentos de la democracia y de los Derechos Humanos.

Teniendo en cuenta las situaciones particulares de los alumnos internos en nuestro $\mathrm{CP}$, el objetivo fundamental es el número 4, dado que la educación en la tolerancia y el respeto puede fomentar una adecuada reinserción social y el aumento de las perspectivas ciudadanas (Salamanca, 2016, p. 162).

Respecto a la metodología, resulta clave establecer desde el primer momento del curso la sinergia adecuada entre docentes y alumnos que se base en el mutuo respecto, procurando crear un clima que favorezca el aprendizaje. Trabajar la convivencia en el aula como estrategia educativa da sus frutos a largo plazo; de esta forma, se pueden plantear actividades como la de la comunicación. Se debe recordar que se está planteando realizar una actividad didáctica que simula una comisión de investigación para su posterior juicio, dentro de un centro penitenciario; sin embargo, cuando se planteó la realización de la actividad, los alumnos no se mostraron reticentes, sino muy motivados. Además, tal y como vamos a desarrollar a continuación, las estrategias metodológicas basadas en el aprendizaje activo resultan eficazmente motivadoras en este contexto.

Finalmente, la temporalización de la actividad estaba propuesta para el mes de abril, siendo programada en tres sesiones de dos horas cada una: una primera, donde se desarrollaba la primera actividad, de investigación y determinación de responsabilidades; una segunda sesión, donde se dramatizaba un resumen de los juicios de Núremberg; y una tercera, donde se realizababa un debate discutiendo los resultados de la actividad, culminando con la proyección de la película Núremberg. 
Los instrumentos de evaluación se establecieron mediante la confección de un registro de la actividad diaria, donde se graduaba el nivel de implicación de los alumnos; por otro lado, las producciones escritas sobre el razonamiento a la hora de establecer responsabilidades, presentar pruebas y determinar penas; y, finalmente, la capacidad de expresión oral para poder exponer en conjunto todos los procesos de la actividad. A continuación, se describe la propuesta de actividades que iban a ser realizadas.

\section{Actividad 1: la comisión para el juicio}

Como punto de partida se deben establecer con precisión los contenidos referentes a la Segunda Guerra Mundial, al diseño del Holocausto y al final de dicha contienda. Partiendo de los conocimientos previos trabajados en clase, los alumnos están en disposición de finalizar los contenidos del bloque mediante estas actividades.

Para la primera, se dispone de una sesión de dos horas. Los objetivos didácticos que se pretenden se centran en la capacidad de análisis y comprensión de las responsabilidades tanto de personas como de organizaciones; de esta forma, se procura simular la elección de responsables de las matanzas realizadas durante el régimen nazi.

Previamente se conforman grupos reducidos de alumnos, en este caso se había pensado en tres grupos de cuatro alumnos cada uno, de manera que cada uno representará una comisión aliada. En este punto es clave comprender que los alumnos no pueden representar fielmente los modelos e intenciones políticas y jurídicas de cada una de las comisiones reales de fiscales, por lo tanto, no importa demasiado la nacionalidad de la comisión.

El objetivo de cada una de las comisiones es determinar la imputación y el grado de responsabilidad de individuos y organizaciones en los crímenes cometidos. Como material para llevar a cabo las indagaciones, se aportará por parte del profesor una pequeña ficha bibliográfica de cada uno de los posibles acusados, donde aparecerá su filiación orgánica al régimen nazi, así como datos relevantes en el proceso de la toma de decisiones en el Holocausto. Se repartirán unas 50 fichas biográficas, que incluyen las de los 22 acusados de los procesos, además de otros personajes del III Reich. 


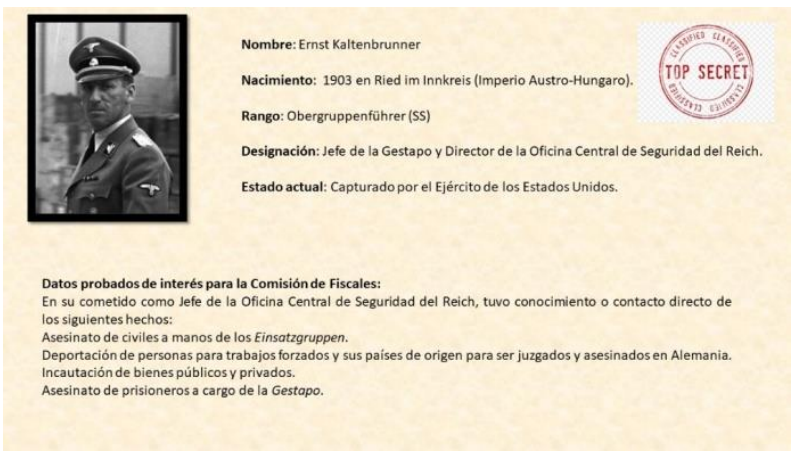

Figura 1: Ejemplo de ficha biográfica de Ernst Kaltenbrunner. (Fuente: elaboración propia).

En segundo lugar, deberán decidir qué cargos se le imputan a cada uno de los personajes incluidos en las listas. La lista de cargos es breve y se puede resumir en esta ficha:

Cargos presentados por la comisión de fiscales:

Crímenes contra la paz

Asesinato

Deportación

Tortura

Apropiación de bienes

Conspiración para asesinato, deportación y tortura

$(\ldots)$

Tabla 1. Cargos generales para los acusados. (Fuente: Obery, R., 2006, y elaboración propia).

Uno de los resultados que se esperan, además de considerarse un objetivo perseguido como comentaremos más adelante, es que los alumnos determinen que el Holocausto supuso un crimen sin parangón y que para ello se debió realizar la tipificación de un delito nuevo: el genocidio y los crímenes contra la Humanidad.

Una vez conformada la lista de acusados con sus respectivos cargos, los alumnos deberán justificar y argumentar las razones. Para finalizar la actividad, se procurará establecer un debate entre ellos sobre el proceso de simulación de la comisión. 


\section{Actividad 2: el juicio de Núremberg}

Se estima en otra sesión de dos horas de duración. La actividad procura dramatizar el juicio a los dirigentes nazis, por lo que en primer lugar se repartirán roles. Dadas las circunstancias de los alumnos internos en un CP, se considera como mejor opción que sea el profesor quien designe los papeles de los diversos acusados.

Las principales pruebas que presentará la acusación estarán en un dossier donde se recogen las diversas actuaciones de los acusados; para ello usarán el material del tema correspondiente a la Segunda Guerra Mundial, donde encontrarán la información pertinente. Dicho dossier incluirá fotografías, mapas y material diverso sobre los campos de exterminio nazis.

El reparto de roles y sus funciones queda determinado de la siguiente forma:

\begin{tabular}{|l|l|}
\hline Roles & Asignación y funciones. \\
\hline Acusados & $\begin{array}{l}\text { Profesor. Deberá presentar argumentos } \\
\text { para la defensa. } \\
\text { Opcional: un alumno representará el } \\
\text { papel de abogado defensor. }\end{array}$ \\
\hline Fiscales. & $\begin{array}{l}\text { Grupos de alumnos. Aportarán pruebas } \\
\text { para ejercer la acusación. Tratarán de } \\
\text { desmontar los argumentos de la } \\
\text { defensa. }\end{array}$ \\
\hline Jueces. & $\begin{array}{l}\text { Alumnos (3). Organizarán el orden del } \\
\text { día del juicio y su secuencia. Tomarán } \\
\text { nota de las acusaciones y las pruebas. } \\
\text { Finalmente dictarán las sentencias } \\
\text { correspondientes. }\end{array}$ \\
\hline
\end{tabular}

Tabla 2: Asignación de funciones en la representación del juicio. (Fuente: elaboración propia).

Una vez determinadas las acusaciones y los hechos probados, se debatirá la sentencia por parte del conjunto de alumnos. Para finalizar, se entregará una memoria 
razonada con la lista de acusados y sus sentencias, que irán desde la pena de muerte hasta la libertad.

\section{Actividad final: recapitulación, debate y visionado de la película Núremberg}

Como actividad de cierre, se proponen una serie de elementos de recapitulación, un debate final donde los alumnos evalúen las conclusiones que puedan extraer del desarrollo del proceso. A este debate se acompañará la información histórica de lo que aconteció en los juicios de Núremberg, con el objetivo de comparar las decisiones tomadas con las sentencias reales; y se propondrán dos temas laterales que pueden dar mucho juego en la discussion: ¿de qué manera se encajarían las sentencias de Núremberg en la España franquista, que había apoyado a los países del Eje? (Vilanova, 2004); y, ¿ha rentabilizado el colectivo judío, tanto política como económicamente, el Holocausto? (Finkelstein, 2014).

Como acto final, dado que el cine es un recurso óptimo en la enseñanza de la Historia y la educación en valores (Ambrós y Breu, 2007; y Pantoja y Moroño, 2019), se propone el visionado de la película Núremberg (Simoneau, 2000). Se ha seleccionado esta obra por ser una de los más recientes sobre el caso y su narración fílmica puede adaptarse mejor al visionado por parte de los alumnos; no se descarta la opción de proyectar, como alternativa, la película ¿Vencedores o vencidos? (Kramer, 1961); quizás menos atractiva apara el alumnado de hoy en día, por ser en blanco y negro, pero con un contenido histórico veraz (Finkelstein, 2014, p. 24).

\section{Consideraciones finales}

Como punto final a esta propuesta de actividad didáctica, debemos analizar de forma apriorística los resultados que se esperaban, así como las dificultades que sin duda se hubieran encontrado en su desarrollo.

La primera dificultad que puede plantear la actividad es la actividad misma, ya que todos los alumnos han sido sometidos a un juicio donde se ha fallado su culpabilidad. Esta misma dificultad puede convertirse en una ventaja, pues el alumno, al alterar su rol, puede comprender mejor la necesidad de no cometer crímenes; es posible que empatice con las víctimas, máxime analizando los peores crímenes cometidos en la 
Historia; y sus nociones de justicia particular pueden verse influidas. De hecho, cuando se propuso realizar la actividad en los inicios del curso, generó mucha expectación entre los alumnos. Además, cabe resaltar que, en el entorno educativo penitenciario, las actividades docentes que sean diferentes a una enseñanza de corte tradicional son muy bien recibidas: no solo sacan de la monotonía al alumnado, como ya se ha dicho, sino que además, al hacerles partícipes de los procesos de toma de decisiones, se favorece el aprendizaje de conceptos históricos complejos.

Respecto a las actividades propuestas, se espera que los alumnos duden a la hora de realizar acusaciones a diversos integrantes del III Reich, dado que la defensa alegará un argumento similar al que alegaron en el propio juicio: eran piezas de un engranaje y solo cumplían órdenes. Ante esta tesitura, los alumnos deben considerar la actuación moral de los individuos y las consecuencias de las acciones que tomaron durante la guerra, especialmente sobre aquellos nazis que estuvieron implicados en el Holocausto.

Los juicios de Núremberg fueron un primer paso, fueron precursores; más de medio siglo después, dado que un sistema tarda generaciones en construirse y ponerse al servicio del común y con la implicación de numerosas instituciones y personas, la Corte Penal Internacional intenta velar porque no vuelvan a suceder crímenes de este calibre, se acabe con la impunidad y los afectados accedan a una justicia judicial que, si no la han encontrado en su país, la hallarán a la sombra de la humanidad como ciudadanos del mundo (Valencia, 2000, p. 90).

\section{Agradecimientos}

Este trabajo ha sido realizado en el marco del Grupo de investigación ARGOS (Gobierno de Aragón / FEDER, UE) y del Instituto Universitario de Investigación en Ciencias Ambientales de Aragón (IUCA-Universidad de Zaragoza).

\section{Referencias}

Ambrós, A. y Breu, R. (2007). Cine y educación. Graó.

Arendt, H. (1999). Eichmann en Jerusalén: un estudio sobre la banalidad del mal. Editorial Lumen. 
Beatriz Acín, A. (2013). La educación secundaria de adultos en la actualidad. Un estudio comparado entre Córdoba (Argentina) y Cataluña (España). Universitat de Barcelona. (Tesis doctoral).

Boudrias, M. y McDougall, I. (productores) y Simoneau, Y. (director) (2000). Núremberg. [Cinta cinematográfica].

Finkelstein, N. G. (2014). La industria del Holocausto. Reflexiones sobre la explotación del sufrimiento judío. Ediciones Akal.

Gobierno de Aragón (2019). Orden ECD/1693/2019.

Gray, M. (2015). Teaching the Holocaust. Practical approaches for ages 11-18. Routledge.

Hayes, P. y Roth, J. K. (2010). The Oxford Handbook of Holocaust Studies. Oxford University Press.

Kramer, S. (productor y director). (1961). ¿Vencedores o vencidos? [Cinta cinematográfica].

Overy, R. (2003). Interrogatorios. El Tercer Reich en el banquillo, Tusquets.

Pantoja Chaves, A. y Moroño Díaz, A. (2019). El cine como recurso en la enseñanza de la Historia. En A. M. Hernández Carretero (Coord.), Estrategias y recursos didácticos para la enseñanza de las Ciencias Sociales (pp. 125-153). Ediciones Pirámide.

Salamanca García, L. (2016). Intervención educativa en prisión: memoria y deseo. Revista de Educación Social, 22, 159-173.

Temon, Y. (1995). El Estado criminal: los genocidios en el siglo XX. Editorial Península, 1995.

Valencia Villa, H. (2000). El genocidio y los crímenes de lesa humanidad ante la Corte Penal Internacional. Revista de Estudios Sociales, 7, 85-90.

Vilanova i Vila-Abadal, F. (2004). Nurembergs. Lectures dels processos contra criminals de guerra a la Barcelona franquista. Fundació Pi i Sunyer. 